\title{
A Comparison between Three-Dimensional Conformal Radiotherapy, Intensity-Modulated Radiotherapy, and Volumetric-Modulated Arc Therapy Techniques for Stereotactic Body Radiotherapy of Lung Tumors
}

\author{
Ganesh Narayanasamy, Drew Granatowicz, Diana Baacke, Ying Li, Alonso Gutierrez, \\ Niko Papanikolaou, Sotirios Stathakis \\ Department of Radiation Oncology, University of Texas Health Science Center at San Antonio, San Antonio, USA \\ Email: stathakis@uthscsa.edu
}

Received 24 January 2015; accepted 28 March 2015; published 7 April 2015

Copyright (C) 2015 by authors and Scientific Research Publishing Inc.

This work is licensed under the Creative Commons Attribution International License (CC BY). http://creativecommons.org/licenses/by/4.0/

(c) (i) Open Access

\section{Abstract}

Introduction: The aim of this study is to dosimetrically compare 3D CRT, IMRT, and VMAT techniques that employ coplanar and non-coplanar beams for the SBRT of lung tumors. Methods and Materials: Nine $(n=9)$ consecutive SBRT lung patients with ten tumor sites who were previously treated at our institution were selected for this study. Six $(n=6)$ treatment plans were created for each PTV: 1 coplanar and 1 non-coplanar 3D CRT, IMRT, and VMAT such that $98 \%$ of PTV received $100 \%$ of prescription dose, Rx of $50 \mathrm{~Gy}$ in 5 fractions. The data collected from each plan included the conformity index, $\mathbf{R}_{50 \%}$, and homogeneity index of the target as well as the volume of normal tissue irradiated by $5 \mathrm{~Gy}, 25 \mathrm{~Gy}$ isodose lines, $V_{5}$ and $V_{20}$ of total lung, and maximum dose to organs at risk. Beam angles and arc lengths were chosen in order to achieve the lowest total lung $V_{5}, V_{20}$ and $R_{50 \%}$ values. Results: According to the observed data, the mean total lung $V_{5}$ and $V_{20}$ values were lowest for the non-coplanar VMAT plans, but were not statistically different from the other planning techniques. Conformity values were similar for the IMRT and VMAT plans, and significantly lower than the 3D CRT plans. $\mathbf{R}_{50 \%}$ values were lowest for the VMAT plans and significantly lower than both IMRT and 3D CRT plans in both coplanar and non-coplanar beam arrangements. However, dose homogeneity in the PTV is significantly higher in the IMRT coplanar plans than the corresponding 3D CRT and SmartArc (SA) coplanar plans. Coplanar VMAT plans were able to produce a significant $35.5 \%$ reduction in the maximum cord dose than coplanar 3D CRT plans (pvalue $<\mathbf{0 . 0 3}$ ). VMAT plans also reduce the volume of the normal tissue irradiated to high doses, 
but the reduction is insignificant. Conclusion: Overall, the VMAT plans were able to produce highly conformal plans. No single planning technique can outclass the others in all of the SBRT planning indices significantly and a thorough review of each patient plan is needed.

\section{Keywords}

\section{Lung SBRT, VMAT, Conformity Index, Homogeneity Index}

\section{Introduction}

Lung cancer is the most common malignancy worldwide and account for the most cancer deaths among both men and women [1]. A widely used treatment modality for inoperable lung cancer, especially early-stage nonsmall cell lung cancer (NSCLC) is stereotactic body radiation therapy (SBRT) [2]. Small sized fields with a sharp penumbra and high fractional dose require the use of advanced immobilization, image-guided localization and more accurate treatment planning procedures. It is common for the majority of stereotactic body radiation therapy (SBRT) of lung tumors to be treated with Intensity Modulation Radiation Therapy (IMRT) [3]. The increased use of SBRT has created the need to evaluate various treatment modalities available including threedimensional conformal radiotherapy (3D CRT), IMRT and the relatively new volumetric-modulated arc therapy (VMAT) [4]. SmartArc (SA) is one version of VMAT that modulates the intensity of the beam as it rotates around the patient. SA delivery method in combination with inverse planning has the ability to produce highly conformal dose distributions while using greatly reduced treatment times [5]. A few reports have demonstrated that SA can deliver highly conformal plans for SBRT of lung [6]-[8]. RapidArc (RA) is another novel implementation of VMAT that has been reported to have superior target dose conformity using less treatment time [3] [8]. The aim of this study is to dosimetrically compare 3D CRT, IMRT, and SA techniques that employ coplanar and non-coplanar beam arrangements for SBRT of lung tumors.

\section{Methods and Materials}

\subsection{Planning}

Nine $(\mathrm{n}=9)$ consecutive SBRT lung patients previously treated at our institution were selected for this study including a patient with bilateral targets. Patients were simulated supine in the SBRT Pro-Lok immobilization device (CIVCO Medical Solutions, Orange City, IA) with abdominal compression. Patients underwent a 4D-CT scan using the Real-time Position Management (RPM) Respiratory System (Varian Medical Systems, Palo Alto, CA) on a GE LightSpeed 16-slice CT scanner (GE Healthcare, Waukesha, WI). The 4D-CT images were used to generate the internal target volume (ITV). Plans were created for treatment on a 23EX linear accelerator (LINAC) (Varian Medical Systems, Palo Alto, CA) using a 6MV photon beam.

For treatment planning, the ITV was expanded by $5 \mathrm{~mm}$ to generate the planning target volume (PTV). The PTV volumes ranged from 6.46 cc to 40.45 cc with locations in the lung as shown in Table 1 . The prescription dose (Rx) of $50 \mathrm{~Gy}$ is delivered in 5 fractions. The organs at risk (OAR) include the lungs, spinal cord, aorta, heart, trachea, liver, and stomach. The dose constraints are based on the TG101 report for a 5 fraction SBRT [4]. Highlights of the dose constraint from the report are shown in Table 2. Tissue heterogeneity correction and 2 $\mathrm{mm}$ dose grid spacing were used in planning. Care was taken to ensure that the higher isodose lines or hotspots occur within the PTV and not in adjacent normal tissue.

Pinnacle ${ }^{3}$ treatment planning system's (Philips Medical System, Andover, MA) collapsed cone convolutionsuperposition (CCCS) algorithmwas used in creating the plans. Six $(n=6)$ treatment plans were created for each case: 1 coplanar (C) and 1 non-coplanar (NC) 3D CRT, IMRT, and SA and normalized such that the Rx covers 98\% of the PTV. While the 3D CRT and IMRT plans consisted of 7 non-opposing fields, the SA-C plans had 2 coplanar arcs of $360^{\circ}$ in the clockwise and counter-clockwise direction. The SA-NC plans consisted of 2 shortened arcs with $10^{\circ}-15^{\circ}$ couch angles and gantry rotation spanning $160^{\circ}-220^{\circ}$, thereby avoiding the contralateral lung. The IMRT and SA plans used identical dose-volume constraints for optimization. Direct machine parameter optimization (DMPO) feature was used during optimization thereby eliminating the need to convert the 
Table 1 . The size and location of the ten $(n=10)$ PTVs used in this study.

\begin{tabular}{ccc}
\hline Case \# & PTV Volume (cc) & Location \\
\hline 1 & 6.46 & Superior Left Lung \\
2 & 9.4 & Superior Right Lung \\
3 & 12.74 & Middle Right Lung \\
4 & 13.93 & Superior Left Lung \\
5 & 15.95 & Superior Right Lung \\
6 & 22.33 & Middle Right Lung \\
7 & 25.3 & Superior Left Lung \\
8 & 34.67 & Superior Right Lung \\
9 & 38.36 & Superior Left Lung \\
10 & 40.45 & Middle Left Lung
\end{tabular}

Table 2. Highlights of the TG-101 OAR dose constraints for a 5-fraction SBRT of lung tumor.

\begin{tabular}{ccc}
\hline OAR & Max critical volume (cc) & Threshold dose (Gy) \\
\hline Total Lung & 1500 & 12.5 \\
Total Lung & 1000 & 13.5 \\
Liver & 700 & 21 \\
Spinal cord & $<0.35$ & 23 \\
Esophagus & $<5$ & 19.5 \\
Heart & $<15$ & 32 \\
Trachea & $<4$ & 16.5 \\
Aorta & $<10$ & 47 \\
Stomach & $<10$ & 18 \\
\hline
\end{tabular}

beam fluence generated to deliverable dose. Following optimization, a final dose calculation was performed using CCCS algorithm at $2 \mathrm{~mm}$ dose grid spacing.

\subsection{Planning Criteria}

Achieving a high degree of target conformity in SBRT lung tumors while sparing the OARs can be quite complex. Plan quality metric for the target includes the ICRU Conformity index (CI) [9], $\mathrm{R}_{50 \%}$, and homogeneity in$\operatorname{dex}(\mathrm{HI})$. CI is defined as:

$$
\mathrm{CI}=100 \% \text { Isodose Volume/PTV Volume }
$$

The closer the value of CI to 1.0 , the better would be the dose conformity. $\mathrm{R}_{50 \%}$ index is an indication of the low dose spillage and is defined as:

$$
\mathrm{R}_{50 \%}=50 \% \text { Isodose Volume/PTV Volume }
$$

Lower $\mathrm{R}_{50 \%}$ ratio indicates greater dose falloff and better dose conformity around the PTV. One way to calculate the homogeneity of the target dose distribution uses the difference in doses delivered to $2 \%$ and $98 \%$ of the volume of PTV represented as $\mathrm{D}_{2 \% \mathrm{~T}}$ and $\mathrm{D}_{98 \% \mathrm{~T}}$, respectively and the mean dose to the PTV $\left(\mathrm{D}_{\text {Mean }}\right)$.

$$
\mathrm{HI}=\left(\mathrm{D}_{2 \% \mathrm{~T}}-\mathrm{D}_{98 \% \mathrm{~T}}\right) / \mathrm{D}_{\text {Mean }}
$$


Values of HI closer to 0 indicate greater dose homogeneity within the volume of PTV while large values indicate more heterogeneous dose distribution. Target coverage depends on the fractional volume of the PTV receiving the Rx dose and is defined as:

$$
\text { Coverage }=\text { Volume of PTV covered by Rx isodose curve } / \text { PTV volume }
$$

With respect to OARs, the maximum dose to the spinal cord, aorta, heart, trachea, liver, and stomach were tracked. The percent volume of total lung that received $5 \mathrm{~Gy}\left(\mathrm{~V}_{5}\right)$, $20 \mathrm{~Gy}\left(\mathrm{~V}_{20}\right)$ as well as the normal tissue irradiated by 5 Gy and 25 Gy isodose line were recorded. Beam angles and arc lengths were chosen for the six treatment plans in order to meet $98 \%$ coverage of the PTV and the least value of total lung $V_{5}$ and $V_{20}$ and $R_{50 \%}$. The data were extracted from the plan dose volume histogram (DVH) and were averaged for each patient and for each planning technique. The isodose distribution and the corresponding DVHs were shown for one representative patient in Figure 1 and Figure 2 respectively. The indices were compared across the six treatment techniques for the same beam configuration using a 2-tailed Student's paired T-test.
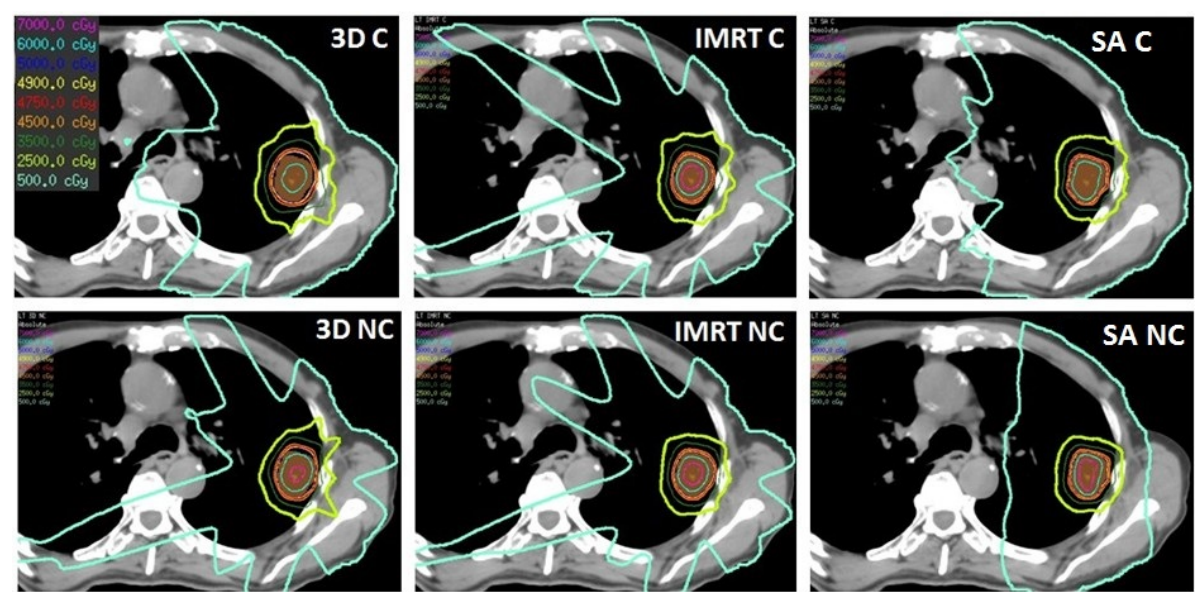

Figure 1. Isodose line comparison for PTV \#4. Top row, left to right: coplanar 3D CRT, IMRT, and SA. Bottom row, left to right: non-coplanar 3D CRT, IMRT, and SA.

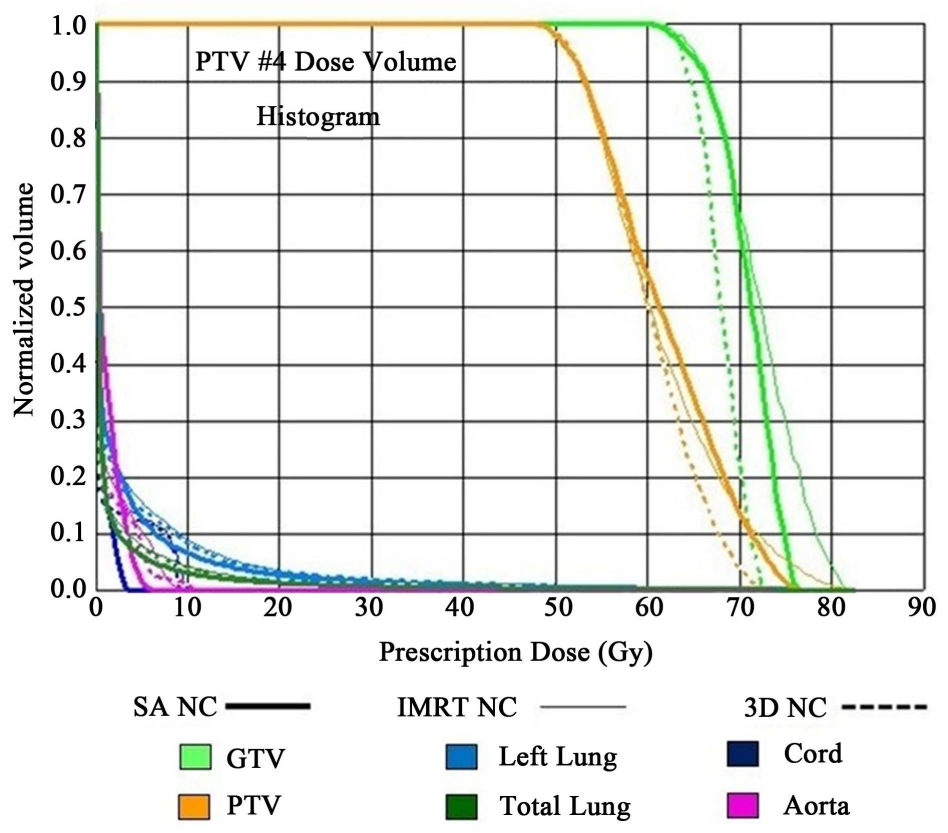

Figure 2. DVH of the GTV, PTV, total lung, left lung, cord, and Aorta for PTV \#4. 


\section{Results}

Prior to analysis, the patient plans are sorted by the volume of PTV, as shown in Table 1 and in all the figures. Equation (1), Equation (2) and Equation (3) were used in tabulation of values of CI, $\mathrm{R}_{50 \%}$ and HI from the 6 treatment plans, respectively. Dose conformity of the PTV coverage could be judged by the value of CI. Ideally, CI should be less than 1.2 and values up to 1.5 are considered as a minor deviation for a clinically deliverable lung SBRT plan, per RTOG 0813. As shown in Figure 3, the measured CI values $<1.5$, with the exception of the 3D CRT coplanar plans of PTV\# 1, 6 in which case CI values $<1.75$.

The low dose spillage depends on the dose falloff gradient beyond the PTV extending into normal tissue and is measured using the $\mathrm{R}_{50 \%}$ parameter. The measured $\mathrm{R}_{50 \%}$ values ranged between 3.4 and 6.8, as shown in Figure 4 . This can be compared against the acceptable $\mathrm{R}_{50 \%}$ values tabulated under 2 categories-no deviation and with minor deviation in Table 1 in RTOG 0813. The generated plans had larger than a minor deviation in the $\mathrm{R}_{50 \%}$ values of 3D CRT coplanar plan in PTVs 4, 5 and for both configuration of 3D CRT plans in PTV\#9.

The target dose homogeneity was measured by the ratio of the difference between doses that irradiate $2 \%$ and 98\% of the PTV and the mean dose to PTV. Shown in Figure 5 are the measured HI values that range between 0.25 and 0.6 .

Shown in Table 3 is a summary of the PTV and OAR dosimetric comparison of the six treatment techniques averaged across patients. The measured data for PTV is plotted in Figure 6. IMRT and SA produced the most conformal plans with mean CI values close to unity (1.04). The mean CI values for coplanar and non-coplanar 3D CRT plans were on average significantly higher by $26 \%$ and $19 \%$ with p-value $<0.01,0.01$ respectively. The CI values of coplanar and non-coplanar SA plans were not significantly different from those of the corresponding IMRT plans.

As shown in Figure 6, the mean $\mathrm{R}_{50 \%}$ values were lowest for the non-coplanar SA plans with a value of 4.19. The mean $\mathrm{R}_{50 \%}$ values for the 3D CRT non-coplanar and IMRT non-coplanar plans were 13\% and 16\% higher,

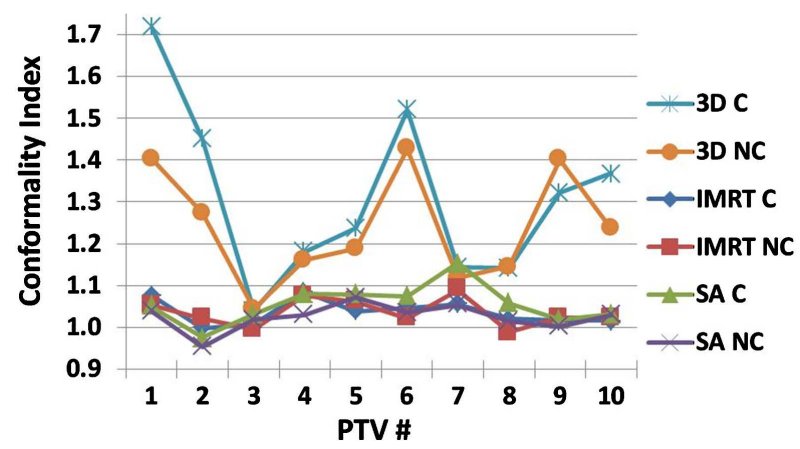

Figure 3. Conformity index for the 6 plans generated for each of the 10 PTVs. Notice that with the exception the 3D CRT coplanar plans of PTV\# 1 and $6, \mathrm{CI}<1.5$.

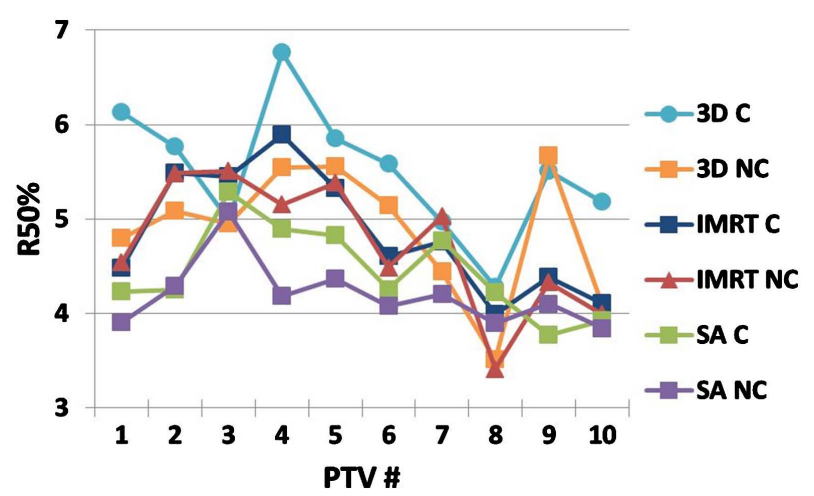

Figure 4. $\mathrm{R}_{50 \%}$ indices for the 6 plans generated for each of the 10 PTVs. 


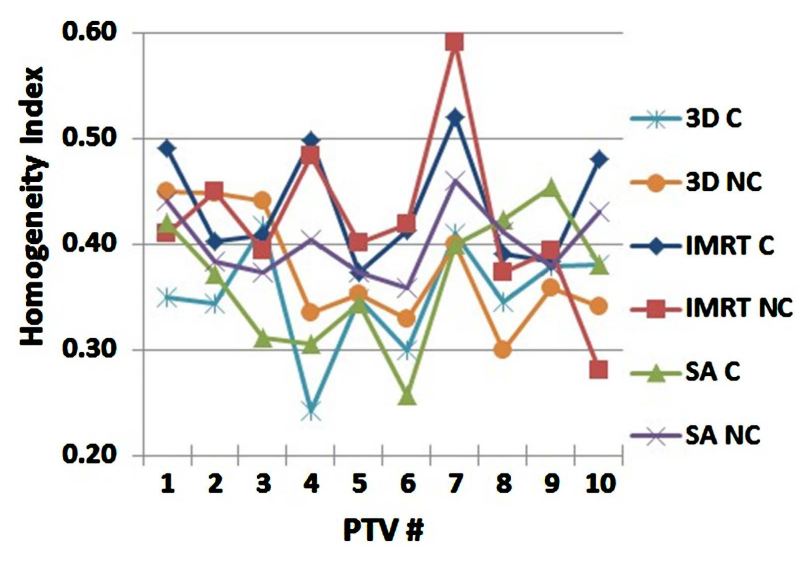

Figure 5. Homogeneity index for the 6 plans generated for each of the 10 PTVs.

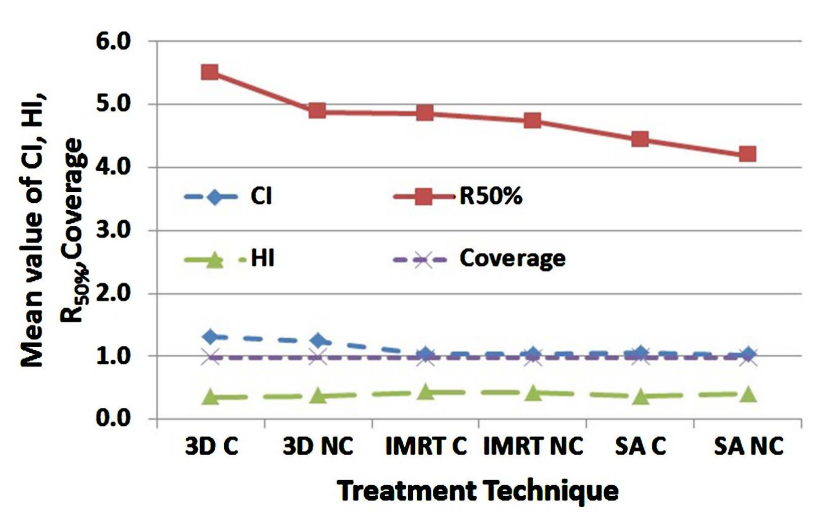

Figure 6. Dosimetric comparison of treatment techniques based on the $\mathrm{CI}, \mathrm{HI}, \mathrm{R}_{50 \%}$ and Coverage values averaged among the 10 patient plans.

Table 3. Summary of dosimetric comparison between the six treatment techniques expressed as mean values \pm standard deviation. " and "indicates statistical significance based on Student's 2-tailed T-test (p-value $<0.05)$ with respect to the corresponding coplanar (C) or non-coplanar (NC) configuration of 3D CRT, IMRT plans respectively.

\begin{tabular}{|c|c|c|c|c|c|c|}
\hline & 3D C & 3D NC & IMRT C & IMRT NC & SA C & SA NC \\
\hline CI & $1.31 \pm 0.21$ & $1.24 \pm 0.13$ & $1.04 \pm 0.03^{*}$ & $1.04 \pm 0.03^{*}$ & $1.05 \pm 0.05^{*}$ & $1.02 \pm 0.03^{*}$ \\
\hline $\mathrm{R} 50 \%$ & $5.50 \pm 0.69$ & $4.88 \pm 0.69$ & $4.85 \pm 0.65^{*}$ & $4.73 \pm 0.70$ & $4.44 \pm 0.48^{*}$ & $4.19 \pm 0.35^{*+}$ \\
\hline $\mathrm{HI}$ & $0.35 \pm 0.05$ & $0.38 \pm 0.06$ & $0.44 \pm 0.05^{*}$ & $0.42 \pm 0.08$ & $0.37 \pm 0.06^{+}$ & $0.40 \pm 0.03$ \\
\hline Coverage & $0.98 \pm 0.0$ & $0.98 \pm 0.0$ & $0.98 \pm 0.0$ & $0.98 \pm 0.0$ & $0.98 \pm 0.0$ & $0.98 \pm 0.0$ \\
\hline Max Cord (Gy) & $11.38 \pm 4.03$ & $6.94 \pm 4.99$ & $10.91 \pm 4.87$ & $6.52 \pm 5.72$ & $7.34 \pm 3.49^{*}$ & $6.07 \pm 3.61$ \\
\hline Max Heart (Gy) & $10.35 \pm 11.58$ & $10.19 \pm 8.9$ & $10.14 \pm 10.25$ & $10.87 \pm 9.26$ & $9.15 \pm 9.48$ & $9.16 \pm 8.79$ \\
\hline Lung V5Gy (\%) & $0.13 \pm 0.04$ & $0.13 \pm 0.04$ & $0.14 \pm 0.04$ & $0.14 \pm 0.05$ & $0.16 \pm 0.06$ & $0.13 \pm 0.05$ \\
\hline Lung V20Gy (\%) & $0.03 \pm 0.02$ & $0.03 \pm 0.01$ & $0.03 \pm 0.01$ & $0.03 \pm 0.01$ & $0.03 \pm 0.02$ & $0.03 \pm 0.02$ \\
\hline
\end{tabular}

respectively. The $\mathrm{R}_{50 \%}$ values of non-coplanar SA plans were significantly different from that of both IMRT non-coplanar and 3D CRT non-coplanar plans with p-value $<0.05$ and $\mathrm{p}$-value $<0.02$, respectively. The $\mathrm{R}_{50 \%}$ values of coplanar SA plans were significantly different from that coplanar 3D CRT plans with p-value $<0.001$, but not significantly different from the coplanar IMRT plans with p-value $>0.10$. 
The mean HI values range between 0.35 and 0.44 for the 6 treatment plans. Dose heterogeneity in the PTV is significantly higher in the IMRT coplanar plans than the corresponding 3D CRT and SA coplanar plans with pvalue $<0.005$ and p-value $<0.05$, respectively.

In the measured OAR data, the quantities analyzed include $\mathrm{V}_{5}, \mathrm{~V}_{20}$ for the total lung, and volumes of normal tissue irradiated by 5 Gy, 25 Gy as well as the maximum dose to spinal cord, aorta, heart, trachea, liver, and stomach. All six treatment techniques were able to meet the OAR dose constraints and the only significant reduction in OAR dose came from coplanar VMAT plans for a 35.5\% reduction in the maximum cord dose than coplanar 3D CRT plans (p-value $<0.03$ ). The maximum dose to other OARs were not significantly different (pvalue $>0.05$ ). The deviations in $\mathrm{V}_{5}$ and $\mathrm{V}_{20}$ of the total lung are negligible among the six treatment techniques, as shown in Figure 7, and the coplanar SA plans had the highest mean $V_{5}$ value of $0.16 \pm 0.06$.

Regarding 5 Gy, 25 Gy isodose volumes of the normal tissue surrounding the target, there is no significant difference among the six treatment techniques. The 3D non-coplanar and SA non-coplanar plans had the lowest mean values of 5 Gy, 25 Gy volume of normal tissue respectively, while the highest values were from the SA coplanar plans and the 3D CRT coplanar plans, as displayed in Figure 8.

\section{Discussion}

Our study compared the 3D CRT, IMRT and SA planning techniques for SBRT of lung tumor using both cop-lanar and non-coplanar beam arrangement. Similar studies and article are available in the literature [3] [7] [10] [11]. While Holt et al. compared the efficacy of coplanar SA against IMRT for SBRT of early stage lung

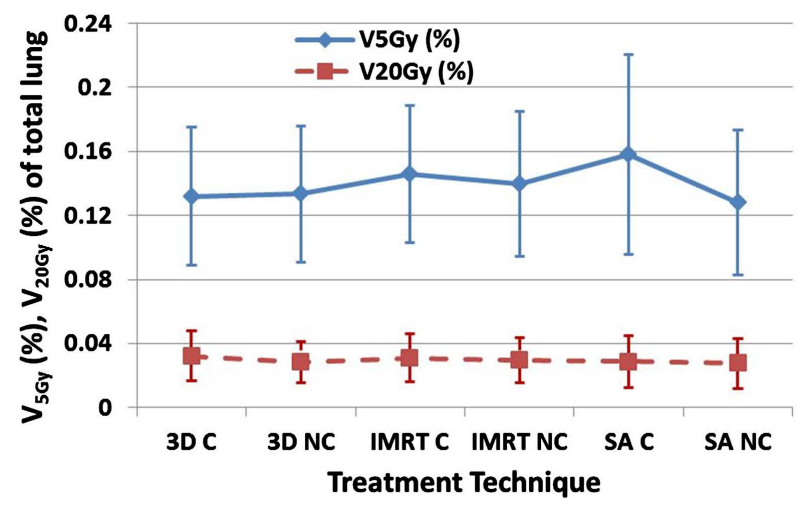

Figure 7. The percentage of total lung irradiated by 5 Gy, 20 Gy isodose lines, shown as mean value \pm 1 standard deviation. Notice that the differences among the 6 treatment techniques are insignificant.

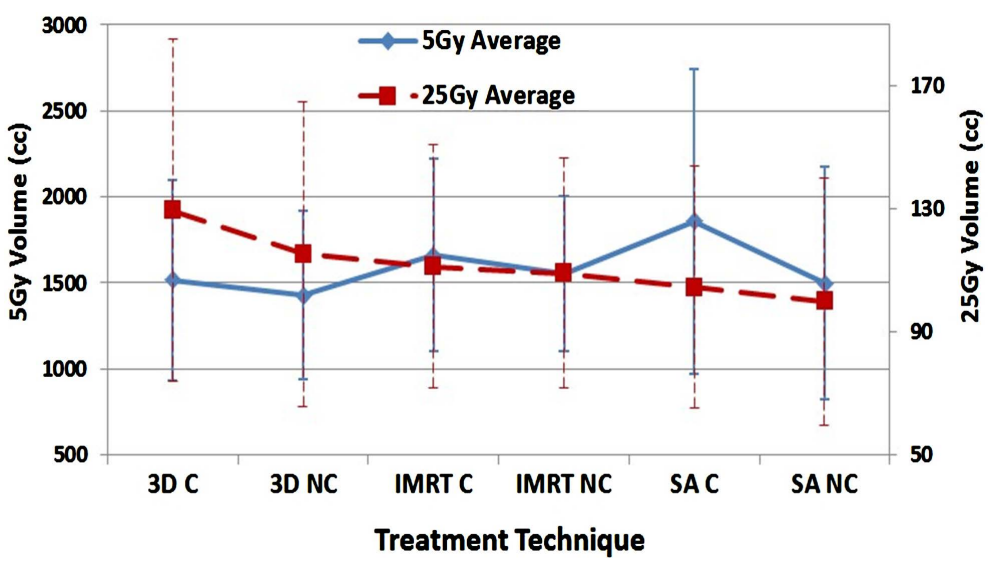

Figure 8. The volume of normal tissue covered by the 5 Gy and 25 Gy isodose lines for the six treatment techniques, shown as mean values \pm 1 standard deviation. 
cancer [7], Bree et al. studied delivery of higher dose to lung tumor using IMRT than that was possible with 3D-CRT [10].

The dose distributions in Figure 1 nicely demonstrate the target dose conformity improvements with both coplanar and non-coplanar IMRT and SA than 3D CRT plans. The dose distribution in non-coplanar SA plan with 2 shorter arcs was slightly more conformal than that in coplanar SA plan with 2 complete arcs, but the improvements in CI are not significant. The conformity of the $100 \%$ isodose lines of the IMRT and SA plans were well within the clinical acceptable limit specified in RTOG 0813 protocol of $\mathrm{CI} \leq 1.2$ and upto 1.5 would be a minor deviation. 2 of the 3D-CRT plans violated the minor deviation specification. For target dose conformity, the IMRT and SA plans scored higher than 3D CRT plans, which concurred with the results from Wu et al. [12]. Values of CIs are comparable with Verbakel et al. [5], although in contrast to the findings in that study, we did not observe any improvement in CIs with SA plans compared with that of non-coplanar IMRT plans. The number and arrangement of beams as well as the width of multileaf collimator might play a role.

Regarding $\mathrm{R}_{50 \%}$, both IMRT and SA plans gave tighter dose distribution than the 3D CRT plans. Specifically, $\mathrm{R}_{50 \%}$ values of non-coplanar SA plans were significantly smaller than that of all other plans. With the exception of 2 cases, $R_{50 \%}$ values of non-coplanar SA plans were the lowest in comparison with other plans. This was expected, as non-coplanar SA spreads out the dose in non-coplanar directions while the dose deposited in the coplanar SA plan is within the plane of the arc. This is consistent with results from other similar studies [7] [8].

Heterogeneity of target dose distribution is higher in IMRT coplanar plans than those of both 3D CRT and VMAT coplanar plans. A nasopharyngeal tumor study reported that the 7 field IMRT plans have significantly more heterogeneous dose distribution than VMAT plans [13].

The maximum cord dose was significantly lower for the SA coplanar plans than the 3D CRT coplanar plans. This is in agreement with the findings of a study on VMAT of spinal cord [14]. The $\mathrm{V}_{5}$ of lung and normal tissue were higher with the SA plans, as expected from the distribution of dose through the plane of the arc, but well below the clinically acceptable levels. This is in line with other lung SBRT studies which had quoted similar increase to varying degrees depending on many factors including the beam arrangement [7]. The dosimetric data for the rest of the OARs were not significantly different.

The type of dose calculation algorithm and the TPS could greatly impact the dosimetric results, especially in the case of heterogeneous media and small field geometry. Pinnacle ${ }^{3}$ features the CCCS algorithm that includes scatter corrections in a heterogeneous media. The dose-calculation algorithm has been shown to result in lower conformity in the lung SBRT plans [15]. Some of the dose differences could be due to the limitations in the beam modeling of low density media in the Pinnacle ${ }^{3}$ CCCS algorithm [16]. Coupled with lack of adequate scatter contribution in the small radiation field sizes that are commonly used in SBRT beams, dose differences were observed near the interface [17] [18].

The comparison between 3D CRT, IMRT and SA in this study is specific to SBRT of lung tumor. Limitations of this study include any systematic error due to the low patient number evaluated in this study. For IMRT, a seven-field configuration and for SA, a two-arc configuration was examined. Alternate planning techniques using more beams could be attempted. However, incorporation of such changes could increase the dosimetric quality and the plan complexity at the cost of treatment time. Long-term complication-free survival and patient outcomes are needed before definite conclusions can be made. It is advisable to analyze treatment plans of each patient to determine which plan will be the most suited. The suitability of the treatment technique could vary depending on the staging, tumor size, location of tumor, vicinity of the OARs as well as the dose tolerance criteria observed.

\section{Conclusion}

In this study, all six treatment techniques were able to deliver conformal SBRT plans while meeting the dose constraints of the OARs. While IMRT and SA plans scored better than 3D CRT plans with regard to dose conformity and dose fall-off, IMRT plans fared better than others in the target dose homogeneity index. SA coplanar plans provided significantly lower maximum dose to the cord. However, no one treatment technique was able to produce a plan with significantly superior plan quality indices over others for any given patient.

\section{References}

[1] Jemal, A., Siegel, R., Xu, J. and Ward, E. (2010) Cancer Statistics. CA: A Cancer Journal for Clinicians, 60, 277-300. 
http://dx.doi.org/10.3322/caac.20073

[2] Videtic, G., Stephans, K., Reddy, C., Gajdos, S., Kolar, M., Clouser, E. and Djemil, T. (2010) Intensity-Modulated Radiotherapy-Based Stereotactic Body Radiotherapy for Medically Inoperable Early-Stage Lung Cancer: Excellent Local Control. International Journal of Radiation Oncology*Biology* Physics, 77, 344-349. http://dx.doi.org/10.1016/j.ijrobp.2009.05.004

[3] Ong, C., Verbakel, W., Cuijpers, J., Slotman, B.J., Lanerwaard, F.J. and Senan, S. (2010) Stereotactic Radiotherapy for Peripheral Lung Tumors: A Comparison of Volumetric Modulated Arc Therapy with 3 Other Delivery Techniques. Radiotherapy \& Oncology, 97, 437-442. http://dx.doi.org/10.1016/j.radonc.2010.09.027

[4] Benedict, S.H., Yenice, K.M., Followill, D., Galvin, J.M., Hinson, W., Kavanagh, B., et al. (2010) Stereotactic Body Radiation Therapy. The Report of the AAPM Task Group 101. Medical Physics, 37, 4078-4101. http://dx.doi.org/10.1118/1.3438081

[5] Verbakel, W., Senan, S., Cujipers, J., Slotman, B.J. and Lagerwaard, F.J. (2009) Rapid Delivery of Stereotactic Radiotherapy for Peripheral Lung Tumors Using Volumetric Intensity-Modulated Arcs. Radiotherapy \& Oncology, 93, 122124. http://dx.doi.org/10.1016/j.radonc.2009.05.020

[6] Brock, J., Bedford, J., Partridge, M., McDonald, F., Ashley, S., McNair, H.A., et al. (2012) Optimizing Stereotactic Body Radiotherapy for Non-Small Cell Lung Cancer with Volumetric Intensity-Modulated Arc Therapy-A Planning Study. Clinical Oncology, 24, 68-75. http://dx.doi.org/10.1016/j.clon.2011.02.003

[7] Holt, A., van Vliet-Vroegindeweij, C., Mans, A., Belderbos, J. and Damen, E.M. (2011) Volumetric-Modulated Arc Therapy for Stereotactic Body Radiotherapy of Lung Tumors: A Comparison with Intensity-Modulated Radiotherapy Techniques. International Journal of Radiation Oncology*Biology* Physics, 81, 1560-1567. http://dx.doi.org/10.1016/j.ijrobp.2010.09.014

[8] Weyh, A., Konski, A., Nalichowski, A., Maier, J. and Lack, D. (2013) Lung SBRT: Dosimetric and Delivery Comparison of Rapid Arc, Tomo Therapy, and IMRT. Journal of Applied Clinical Medical Physics, 14, 3-13.

[9] International Commission on Radiation Units and Measurements (1999) Prescribing, Recording and Reporting Photon Beam Therapy. ICRU Report 62. ICRU Publications, Bethesda.

[10] Bree, I.D., van Hinsberg, M.G. and van Veelen, L.R. (2012) High-Dose Radiotherapy in Inoperable Nonsmall Cell Lung Cancer: Comparison of Volumetric Modulated Arc Therapy, Dynamic IMRT and 3D Conformal Radiotherapy. Medical Dosimetry, 37, 353-357. http://dx.doi.org/10.1016/j.meddos.2011.12.002

[11] Cai, J., Malhotra, H.K. and Orton, C.G. (2014) A 3D Conformal Technique Is Better than IMRT or VMAT for Lung SBRT. Medical Physics, 41, Article ID: 040601. http://dx.doi.org/10.1118/1.4856175

[12] Wu, V.W., Kwong, D.L. and Sham, J.S. (2004) Target Dose Conformity in 3-Dimensional Conformal Radiotherapy and Intensity Modulated Radiotherapy. Radiotherapy \& Oncology, 71, 201-206. http://dx.doi.org/10.1016/j.radonc.2004.03.004

[13] Lee, T., Chao, P., Ting, H., Lo, S., Wang, Y., Tuan, C., Fang, F. and Su, T. (2011) Comparative Analysis of Smart Arc-Based Dual Arc Volumetric-Modulated Arc Radiotherapy (VMAT) versus Intensity-Modulated Radiotherapy (IMRT) for Nasopharyngeal Carcinoma. Journal of Applied Clinical Medical Physics, 12, 158-174.

[14] Wu, Q.J., Yoo, S., Kirkpatrick, J.P., Thongphiew, D. and Yin, F.-F. (2009)Volumetric Arc Intensity-Modulated Therapy for Spine Body Radiotherapy: Comparison with Static Intensity-Modulated Treatment. International Journal of Radiation Oncology*Biology* Physics, 75, 1596-1604. http://dx.doi.org/10.1016/j.ijrobp.2009.05.005

[15] Schuring, D. and Hurkmans, C.W. (2008) Developing and Evaluating Stereotactic Lung RT Trials: What We Know about the Influence of Inhomogeneity Corrections on Dose. Radiotherapy \& Oncology, 3, 21. http://dx.doi.org/10.1186/1748-717X-3-21

[16] Oyewale, S. (2013) Dose Prediction Accuracy of Collapsed Cone Convolution Superposition Algorithm in a Multi-Layer Inhomogenous Phantom. International Journal of Cancer Therapy and Oncology, 1, Article ID: 01016. http://dx.doi.org/10.14319/ijcto.0101.6

[17] Stathakis, S., Esquivel, C., Quino, L., Myers, P., Calvo, O., Mavroidis, P., Gutiérrez, A. and Papanikolaou, N. (2012) Accuracy of the Small Field Dosimetry Using the Acuros XB Dose Calculation Algorithm within and beyond Heterogeneous Media for 6 MV Photon Beams. International Journal of Radiation Oncology*Biology* Physics, 1, 78-87. http://dx.doi.org/10.4236/ijmpcero.2012.13011

[18] Calvo, O.I., Gutiérrez, A.N., Stathakis, S., Esquivel, C. and Papanikolaou, N. (2012) On the Quantification of the Dosimetric Accuracy of Collapsed Cone Convolution Superposition (CCCS) Algorithm for Small Lung Volumes Using IMRT. Journal of Applied Clinical Medical Physics, 13, 43-59. 